\title{
Diffuse mesangial sclerosis in a PDSS2 mutation-induced coenzyme Q10 deficiency
}

\author{
Béla Iványi ${ }^{1}$ - Gábor Z. Rácz ${ }^{2}$ Péter Gál ${ }^{2}$ - Kitti Brinyiczki ${ }^{1}$ - István Bódi ${ }^{3}$. \\ Tibor Kalmár ${ }^{2} \cdot{\text { Zoltán } \text { Maróti }^{2} \cdot \text { Csaba Bereczki }}^{2}$
}

Received: 21 April 2017 /Revised: 21 August 2017 / Accepted: 18 September 2017

(C) IPNA 2017

\begin{abstract}
Background A 7-month-old male infant was admitted because he was suffering from nephrotic syndrome, along with encephalomyopathy, hypertrophic cardiomyopathy, clinically suspected deafness and retinitis pigmentosa, and an elevated serum lactate level.

Methods Coenzyme $\mathrm{Q}_{10}$ supplementation was started because of the clinical suspicion of primary $\mathrm{CoQ}_{10}$ deficiency. Despite intensive efforts, he passed away 4 weeks after admission.

Results The results of genetic tests, available postmortem, explored two hitherto undescribed mutations in the PDSS2 gene. Both were located within the polyprenyl synthetase domain. Clinical exome sequencing revealed a heterozygous missense mutation in exon 3, and our in-house joint-analysis algorithm detected a heterozygous large 2923-bp deletion that affected the 5 prime end of exon 8 . Other causative defects in the $\mathrm{CoQ}_{10}$ and infantile nephrosis-related genes examined were not found. A postmortem histological, immunohistochemical, and electron microscopic evaluation of the glomeruli revealed collapsing-sclerosing lesions consistent with diffuse mesangial sclerosis. The extrarenal alterations included hypertrophic cardiomyopathy and diffuse alveolar damage. A
\end{abstract}

Béla Iványi and Gábor Z. Rácz contributed equally to this study and should be considered joint first authors

Béla Iványi

ivanyi.bela@med.u-szeged.hu

1 Department of Pathology, Faculty of Medicine, University of Szeged, Állomás u. 1, Szeged 6725, Hungary

2 Department of Pediatrics and Pediatric Health Center, Faculty of Medicine, University of Szeged, Szeged, Hungary

3 King's College Hospital, London, UK histological evaluation of the central nervous system and skeletal muscles did not demonstrate any obvious abnormality. Conclusions Until now, the clinical features and the mutational status of 6 patients with a PDSS2 gene defect have been reported in the English literature. Here, we describe for the first time detailed kidney morphology features in a patient with nephrotic syndrome carrying mutations in the PDSS2 gene.

Keywords $\mathrm{CoQ}_{10} \cdot P D S S 2$ - Diffuse mesangial sclerosis . Nephrotic

\section{Introduction}

Coenzyme $\mathrm{Q} 10\left(\mathrm{CoQ}_{10}\right)$ deficiency is a rare, clinically heterogeneous autosomal recessive disorder caused by mutation in any of the genes that encode proteins directly involved in the synthesis of the $\mathrm{Q} 10$ coenzyme $\mathrm{CoQ}_{10}$, also known as ubiquinone. Ubiquinone is a small lipophilic molecule located in the inner mitochondrial membrane that is primarily involved in the mitochondrial respiratory chain, where it carries electrons from complexes I and II to complex III. It is also an important antioxidant, a cofactor in the biosynthesis of pyrimidines, and it plays a role in the maintenance of body temperature [1]. The $\mathrm{CoQ}_{10}$ biosynthesis pathway is complex and has not been completely clarified. It requires at least 15 genes in humans, 8 of which (PDSS1, PDSS2, COQ2, COQ4, COQ6, ADCK3, $A D C K 4$, and $C O Q 9$ ) are known to be associated with primary $\mathrm{CoQ}_{10}$ deficiency (MIM 607426) $[2,3]$. Acosta et al. have recently suggested that the genetic defects of the $\mathrm{CoQ}_{10}$ biosynthetic pathway should be classified into three groups [4]. The first group includes PDSS1, PDSS2, COQ2, COQ6, and $A D C K 4$, and they are associated with glomerular renal involvement that manifests as the steroid-resistant nephrotic syndrome. This syndrome may be an isolated finding or it 
may be associated with neurological or systemic manifestations. The second group comprises COQ4, COQ7, and $C O Q 9$, and the main clinical feature is encephalomyopathy. Other manifestations include hypertrophic cardiomyopathy, lactic acidosis, and (if renal involvement is present) tubulopathy. The third group contains $A D C K 3$ and the main clinical feature is cerebellar ataxia.

The PDSS2 gene encodes the decaprenyl diphosphate synthase subunit 2, responsible for the synthesis of the decaprenyl tail of $\mathrm{CoQ}_{10}$. The gene forms a heterotetramer with the PDSS1 gene. In 2000, Rötig et al. reported three siblings with similar symptoms, but with different degrees of severity [5]. They displayed the nephrotic syndrome, neurological impairment (ataxia, dystonia, amyotrophia), retinitis pigmentosa, sensorineural deafness, and cardiomyopathy. Transprenyltransferase deficiency was identified, which was subsequently shown to be caused by a mutation in the PDSS2 gene [6]. $\mathrm{CoQ}_{10}$ therapy was administered in two siblings and this improved their condition. Since then, PDSS2 mutations have been identified in 3 other patients, all with nephrotic syndrome, 1 with compound heterozygous mutations [7], and 2 with homozygous mutations [8]. As renal biopsy was not performed in any of the patients with PDSS2 mutations, the histological changes responsible for the nephrotic syndrome remained undescribed. Here, we report a deceased male infant with nephrotic syndrome, multiorgan failure, and clinical suspicion of primary $\mathrm{CoQ}_{10}$ deficiency. The results of genetic testing, available postmortem, explored two new, unpublished PDSS2 mutations that were considered to be the cause of his fatal disease. The autopsy evaluation of glomeruli revealed diffuse mesangial sclerosis.

\section{Materials and methods}

\section{Clinical history}

Our male infant was the first child of healthy, nonconsanguineous Caucasian parents. He was born after an uncomplicated pregnancy in the 38th gestational week by cesarean section, weighing 3,150 g with an Apgar score of 9/9. He had a variety of mild symptoms from birth. During the newborn screening procedure, his transient otoacoustic emission was abnormal; thus, he was put on the waiting list for brainstem evoked response audiometry scheduled at the age of 6 months. Continuous mild inspiratory stridor warranted direct laryngoscopy, but this revealed a normal anatomy. Weight gain was poor, the stool was never normal, being usually odorous, fluid-like, or mucous. Cystic fibrosis was suspected, but the 18 most frequent mutations in CFTR gene were excluded. At 6 months of age, he underwent a bilateral inguinal hernia operation. Afterward, he developed mild lower leg edema and he was frequently crying. Then he was referred to our hospital at 7 months of age.

Upon admission, he had general edema, muscle hypotonia, mild inspiratory stridor, and global developmental delay. He did not react to auditory stimuli, and we detected no tracking and focusing eye movements. Surprisingly, these symptoms had not been noticed by his parents. Laboratory findings disclosed low serum $\mathrm{Na}(126 \mathrm{mmol} /$ 1), $\mathrm{Cl}(88 \mathrm{mmol} / \mathrm{l})$, hypoproteinemia (total protein $32 \mathrm{~g} / \mathrm{l}$, albumin $16 \mathrm{~g} / \mathrm{l}$ ), dyslipidemia (serum triglyceride, cholesterol, and LDL cholesterol levels were 3.2, 7.6, and $4.6 \mathrm{mmol} / 1$ respectively), anemia, elevated lactate (8$10 \mathrm{mmol} / \mathrm{l})$, and pyruvate $(160 \mu \mathrm{mol} / \mathrm{l})$. The $\mathrm{CK}, \mathrm{LDH}$, GOT, and GPT values were normal. Urinalysis revealed proteinuria; initially $90 \mathrm{mg} / \mathrm{dl}$, it rose to a peak of $1,470 \mathrm{mg} / \mathrm{dl}$. He had mild hypothyreosis and a high aldosterone level, the latter most likely being due to the hyponatremia. The blood pressure measurements revealed moderate hypertension (range $80 / 60-110 / 70 \mathrm{mmHg}$ ). A chest X-ray indicated right-sided hydrothorax and cardiomegaly. Echocardiography revealed pulmonary hypertension and severe left ventricular hypertrophy; the interventricular septum being $18 \mathrm{~mm}$ thick. An electroencephalogram identified nonspecific encephalopathic signs. Ophthalmology and a visual-evoked potential study suggested retinitis pigmentosa, whereas otoneurology found no outer haircell activity.

The markedly elevated serum lactate level, the infantile-onset nephrotic syndrome, the suspected encephalomyopathy, deafness, and retinitis pigmentosa, and the hypertrophic cardiomyopathy, all strongly suggested $\mathrm{CoQ}_{10}$ deficiency. Although we immediately commenced high-dose $\mathrm{CoQ}_{10}$ treatment $(20 \mathrm{mg} / \mathrm{kg} /$ day $)$, the infant's status rapidly deteriorated, probably because of the intractable albumin loss and pulmonary hypertension. Within 2 weeks, he developed kidney failure and anuria, which required hemodialysis. He was put on mechanical ventilation, with inhalative nitrous oxide treatment. In spite of major treatment efforts, he died 1 month after admission.

\section{Genetic analyses}

We performed Illumina TruSight One clinical-exome sequencing, involving the genes related to $\mathrm{CoQ}_{10}$ synthesis (COQ2, COQ4, COQ6, COQ7, COQ8A, COQ8B [ADCK4], COQ9, PDSS1, PDSS2, ADCK1, ADCK2, ADCK5, COQ3, COQ10A, COQ10B, FDXR, FDX2, $A T P X, B R A F, E T F D H)$ and those involved in the pathogenesis of infantile nephrotic syndrome, such as ACTN4, ARHGAP24, CD2AP, CFH, CUBN, DGKE, INF2, ITGA3, ITGB4, KANK1, LAMB2, LMX1B, MYH9, MYO1E, NPHS1, NPHS2, PLCE1, PTPRO, SCARB2, SMARCAL1, TRPC6, and WT1. 
Fig. $1 P D S S 2$ mutations in exon

3 and in exon 8 . a PDSS2

mutation in exon 3. A single nucleotide change c. $485 \mathrm{~A}>\mathrm{G}$ identified in our patient and in the carrier mother ( $\mathrm{R}$ indicating the heterozygous $\mathrm{A} / \mathrm{G}$ state) whereas the father was homozygous for normal c.485A allels. b The 162 His amino acid is conserved among the vertebrae $(\mathrm{H}$ highlighted in red) An alignment showing the relevant part of PDSS2 protein in six vertebrate species. c A heterozygous 2923bp deletion identified in our patient and in the carrier father. The Sanger traces show the deletion breakpoint (indicated by a red arrow) within exon 8 , in the case of the index patient and his father, whereas the unaffected maternal sequence is shown for comparison
A

\begin{tabular}{|c|c|c|c|}
\hline \multicolumn{4}{|c|}{ V161 H162 R163 } \\
\hline $\mathbf{W}$ & GTA & CAT & CGT \\
\hline MT & GTA & CGT & CGT \\
\hline & V16 & R16 & 16 \\
\hline
\end{tabular}

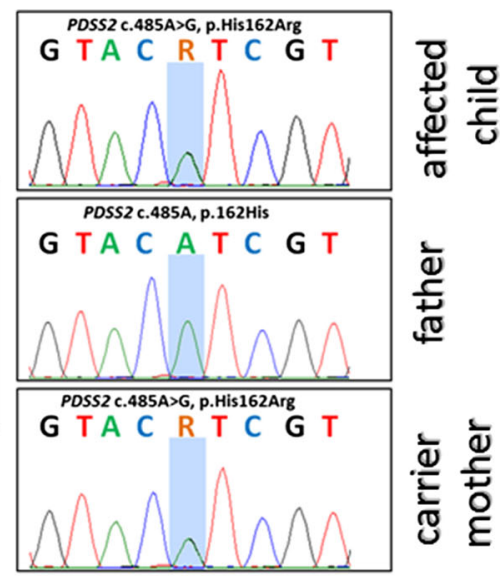

B

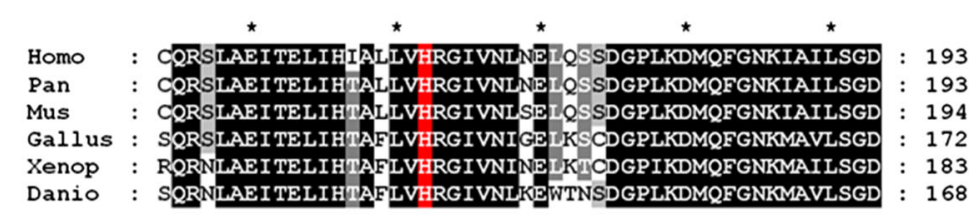

C
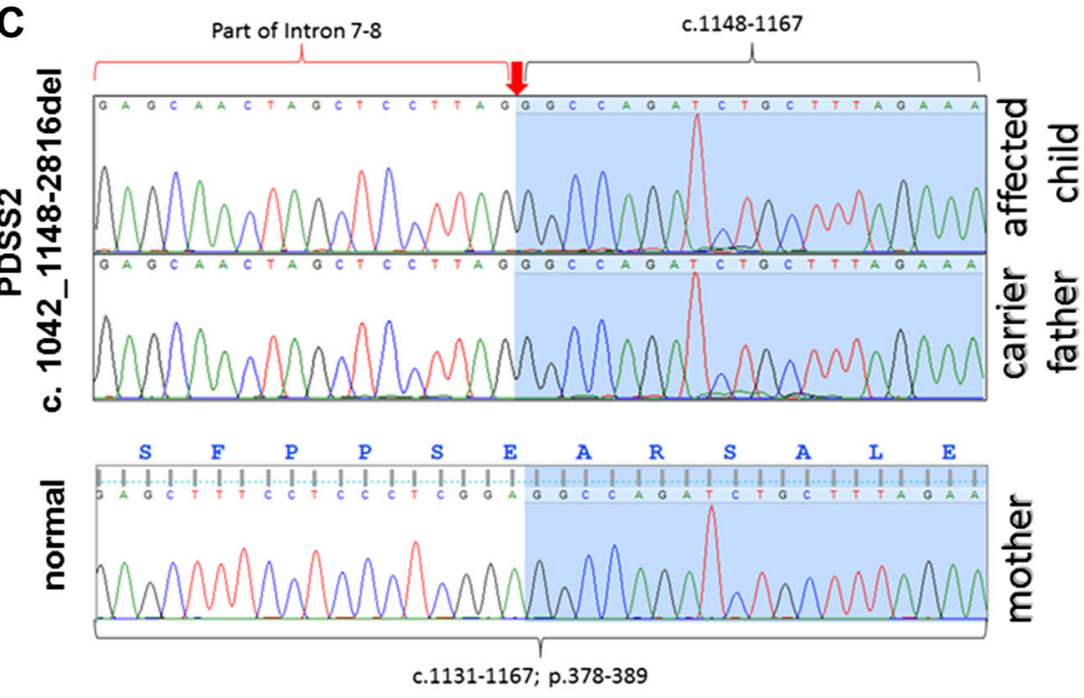

A heterozygous missense mutation in the PDSS2 gene in exon 3 was found that could have caused a defect in mitochondrial ATP production (Fig. 1a). PDSS2 exon 3 c. $485 \mathrm{~A}>\mathrm{G}$, p.His162Arg mutation has a SIFT prediction "deleterious" (0) and a PolyPhen prediction "probably damaging" (1) $[9,10]$. The 162 His amino acid is conserved among the vertebrae (H short for His is highlighted in red in Fig. 1b) and since the PhyloP conservation score is 4.71, this indicates a conserved site [11].

Our database contains the coverage data of 200 TruSight One runs that were performed on the same platform, machine, wetlab protocols, and bioinformatic analysis pipeline. The hybridization protocol used for TruSight One ensures nearly linear target enrichment; thus, the only complications that could arise would be due to GC bias and wetlab errors, which result in significantly altered insert sizes or low coverage. Our inhouse joint-analysis algorithm is based on the following principles:

1. We assessed the wetlab QC values and the overall variability of the coverage profile for each sample to determine whether the sample is suitable for $\mathrm{CNV}$ analysis

2. Using the coverage profile of QC-passed samples for each bed coordinate, the $\log 3$ normalized average coverage and the variance of coverage were calculated using the bedcov algorithm of samtools (version 1.3.1-50-gd02c177) [12]

3. Depending on the average coverage and variance, the regions were classified into normal, null coverage, low 
normalized average coverage categories indicating no $\mathrm{CNV}$, homozygous deletion, heterozygous deletion, and duplication respectively

4. Potential CNV alterations in the disease-specific genes were checked individually

Using our algorithm, we detected a heterozygous 2923-bp deletion that affected a part of the last exon (exon 8) in the PDSS2 gene (Fig. 1c). At the time, we did not find any other pathological alteration in the CoQ10 or nephrotic-related genes in question. We validated both mutations by Sanger sequencing in the patient sample using the following primer pairs (PDSS2_IVS7-Ex8F 5-CTCCAGCCTGGGTG ATAGAG-3, PDSS2_IVS7-Ex8R 5-GCTCCCAATCAACC TCATTC-3, PDSS2-Ex3F 5-TTACAGATCCCAAA CTGCTCA-3, and PDSS2-Ex3R 5-AGCTCCAGCAGCCA ACTAAT-3).

The parental carrier analysis confirmed that the maternal mutation is the NM_020381.3:c.485A > G (p.His162Arg) variant in exon 3 (Fig. 1a) and that the paternal allele is the NM_020381.3:c.1042_1148-2816del, which causes a 107base long deletion of exon 8 (Fig. 1c).

Neither mutation was found in the ExAC database [13] (more than 120,000 chromosomes) nor among 400 population control (Hungarian) chromosomes. Both mutations were located within the highly conserved and essential polyprenyl synthase domain, spanning amino acids (AA) 58-397. The exact molecular effect of the paternal deletion has not been investigated owing to a lack of further cooperation; thus, we use the notation p.? to indicate that an effect at the protein level is expected, but it is not possible to give a reliable molecular prediction of the consequences.

\section{Results}

At autopsy, an external inspection revealed generalized subcutaneous edema with moderate hydrocele. The following body and organ weights were noted: whole body $6,420 \mathrm{~g}$, brain $788 \mathrm{~g}$, heart $44 \mathrm{~g}$, lungs $115 \mathrm{~g}$, kidneys $144 \mathrm{~g}$, liver $250 \mathrm{~g}$, spleen $19 \mathrm{~g}$, and thymus $4 \mathrm{~g}$. The main macroscopic alterations included symmetrically and markedly enlarged kidneys, a hypertrophic left ventricle, and consolidated lung lobes. There was no significant abnormality in the cerebral cortex, the basal ganglia, the brainstem, the cerebellum, or the spinal cord. The ventricles appeared to be slightly dilated. The cranial nerves did not display any change and the eyeballs were normal-sized.

Formalin-fixed paraffin-embedded tissue sections stained with hematoxylin-eosin were made for a histological evaluation of the bone marrow, the thymus, the lymph node, the gastrointestinal tract, the pancreas, the endocrine organs, the skeletal muscles (diaphragm, intercostal muscle, quadriceps), the urinary bladder, and testicles. A detailed neuropathological examination of the central nervous system was performed by the neuropathologist coauthor (IB) on a total of 25 blocks from the brain and the spinal cord. The kidney tissue samples were examined by special stains (PAS, trichrome, and methenamine silver of Jones), immunofluorescence of frozen sections (IgG, IgA, IgM, kappa, lambda, C3, C1q, and fibrinogen), and by electron microscopy. The immunophenotype of glomerular epithelial cells was studied using antibodies against Wilms tumor-1 (WT1; marker of mature podocytes; Cell Marque, Rocklin, CA, USA; clone 6F-H2; an antibody dilution 1:300, pH 6), PAX2 (marker of parietal epithelial cells; Abgent, San Diego, CA, USA; clone EP3251; dilution 1:250, $\mathrm{pH}$ 9), cytokeratins (AE1/AE3 cocktail; Cell Marque; dilution 1:300, $\mathrm{pH}$ 9) and Ki-67 (proliferation marker; Biocare, Pacheco, CA, USA; clone SP6; dilution 1:100, $\mathrm{pH}$ 6) on paraffin sections.

Histologically, the glomeruli in the subcapsular cortex appeared fetal (Fig. 2a). The corpuscles in the deeper cortex were larger and more mature, and exhibited three types of lesions. The first type consisted of an increase in the mesangial matrix, occasionally accompanied by mild mesangial hypercellularity. The second type was characterized by the combination of thickened, sometimes double-contoured, glomerular basement membrane (GBM) and a marked expansion of the mesangium (trichrome: blue, silver: positive) leading to the closure of the capillary tufts. Enlarged podocytes covered the consolidated areas (Fig. 2b-d). The third type, affecting a few glomeruli, manifested in the shrunken solidified glomeruli, covered by a corona of epithelial cells (Fig. 2e). Pseudocrescents or sclerosing capsular adhesions were not observed. The tubuli appeared to be dilated, but cystically dilated profiles were not observed. Signs of acute kidney injury (i.e., mitoses in tubular epithelial cells, distal tubular casts, and granulocytic and erythroid precursors in the vasa recta) were not seen. Mild focal interstitial fibrosis and tubular atrophy accompanied the glomerular alterations (Fig. 2a, b). However, the arteries and arterioles were normal. As regards the immunophenotype of glomerular epithelial cells, the podocytes and the parietal epithelial cells did not react with the AE1/AE3 cytokeratin cocktail (Fig. 2f). The WT1 staining, which diffusely decorated the cytoplasm of podocytes in the subcapsular cortex (Fig. 3a), proved focally negative at sites of capillary consolidation (Fig. 3b). By contrast, some of the podocytes covering the consolidated regions expressed PAX2 positivity (Fig. 3d). The Ki-67-staining in podocytes revealed 0 to 4 signals in a glomerular profile (Fig. 3c). An immunofluorescence examination of the glomeruli proved negative. Six glomeruli were evaluated ultrastructurally. Although the postmortem conditions limited the assessment of the fine structure of the cell organelles, the alterations in the GBM could be unambiguously discerned. Effacement of foot processes was seen in about $70 \%$ of the circumference of 

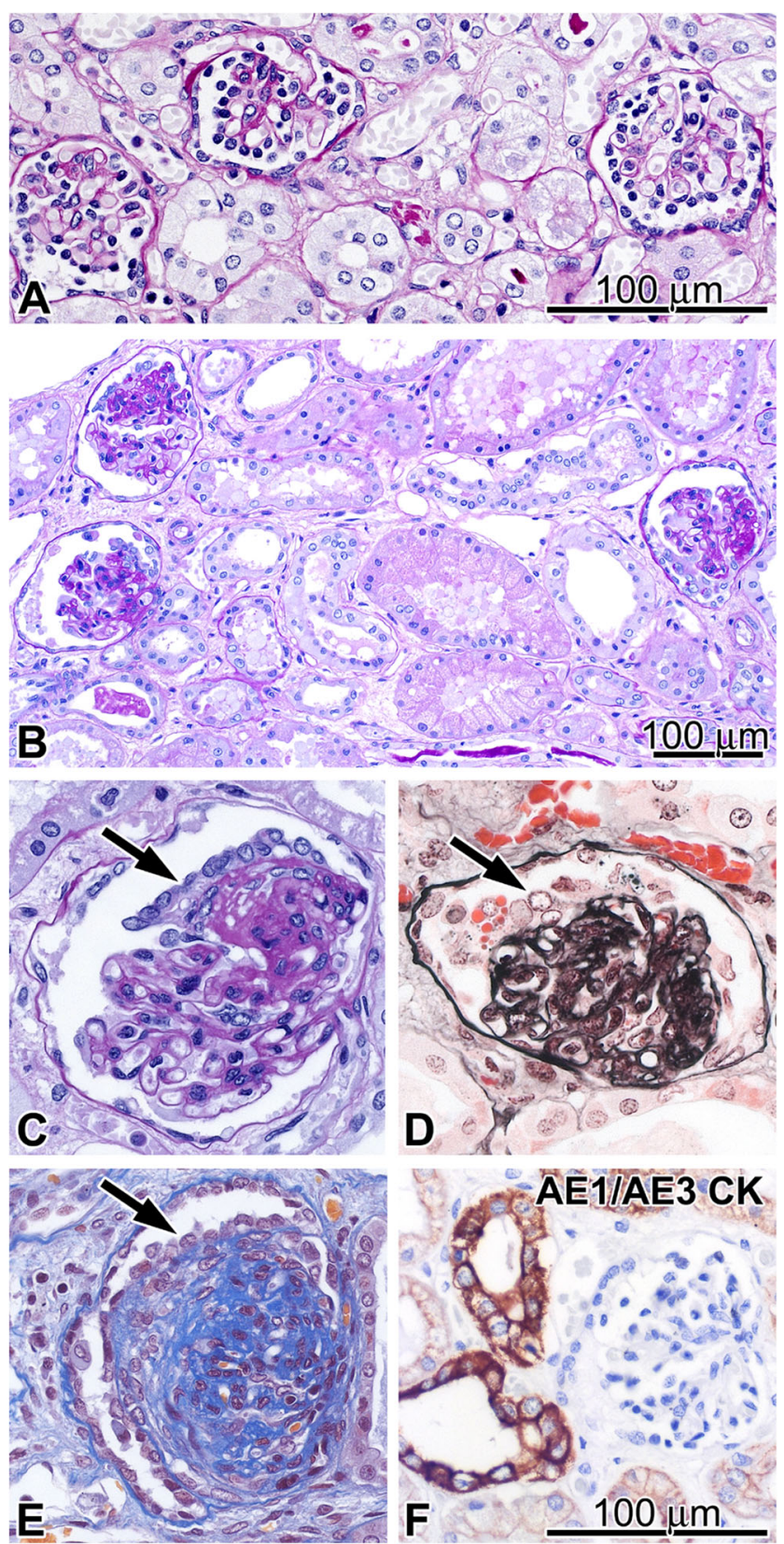

Fig. 2 Histological features of the glomeruli, tubuli, and interstitium in PDSS2 mutation. a Subcapsular cortex: the prominence of closely spaced podocytes gives the glomeruli a fetal appearance. The tubuli and the interstitium display no alteration (PAS). b Glomeruli from the deep cortex: segmental closure of capillary tufts covered by enlarged podocytes. The tubuli and the interstitium do not show any remarkable alteration (PAS). $\mathbf{c}$ and $\mathbf{d}$ Diffuse mesangial sclerosis, the fully developed stage: segmental consolidation of the capillary tufts covered by enlarged podocytes (arrow). (c PAS, d Jones' silver). e Diffuse mesangial sclerosis, the advanced stage: a solidified glomerular profile with the disappearance of glomerular tufts, and a corona of epithelial cells (arrow) (trichrome). $\mathbf{f}$ The podocytes and the parietal epithelial cells do not express AE1/AE3positive cytokeratins

capillary tufts. Capillary tuft consolidation was observed in 2 of the 6 glomeruli. In these sites, the podocytes were enlarged and contained many dense vacuoles, and several layers of
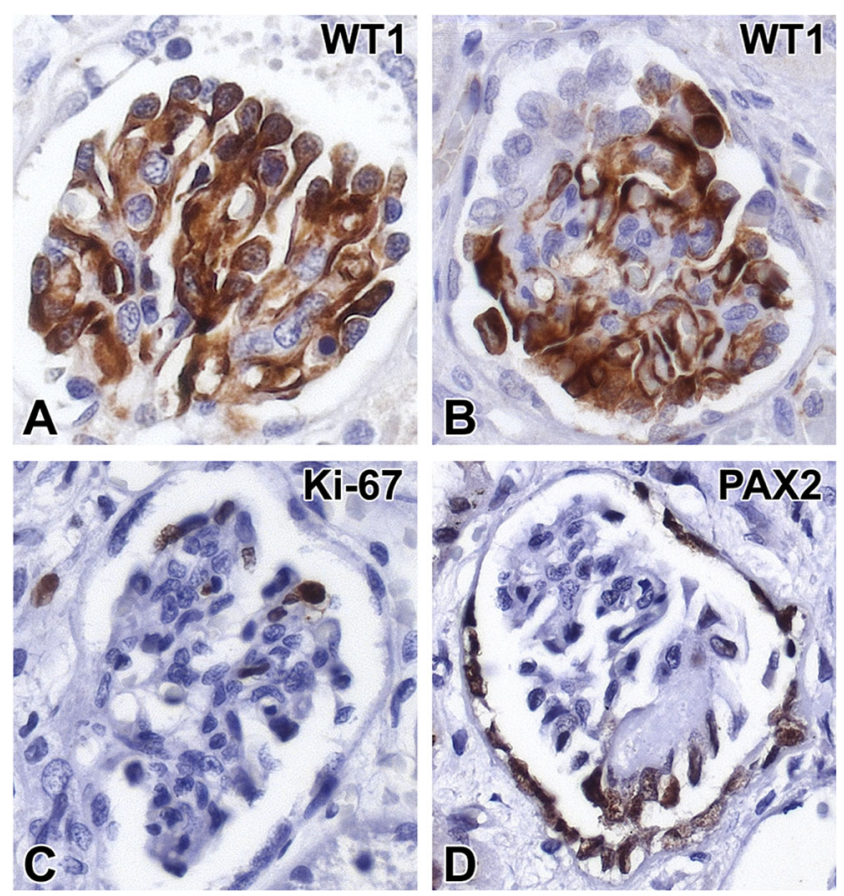

Fig. 3 Dysregulated podocytes in diffuse mesangial sclerosis. a Podocytes in fetal-like glomeruli display uniform cytoplasmic positivity with the WT1 staining. b By contrast, some of the epithelial cells overlying the collapsed capillaries appear to be WT1-negative. c The proliferation marker Ki-67 commonly exhibits nuclear positivity in cells opposite the urinary space. d Nuclear PAX2 positivity in cells covering the sclerosed glomerular segment and in the cells of Bowman's capsule

basement membrane material were deposited in the subepithelial and subendothelial space of the capillary wall (Fig. 4). The mesangial cells were enlarged and were surrounded by an abundant mesangial matrix. Mitochondrial inclusion bodies were not observed in the cells of glomerular and tubular profiles. The glomerular alterations detailed above were consistent with diffuse mesangial sclerosis (DMS).

The cortical lamination, the visual cortex, the optic nerves, and all structures of the brain and brainstem appeared to be histologically normal. A histological evaluation of the left ventricular myocardium revealed pronounced cardiomyocyte hypertrophy, with the periodic acid-Schiff-negative perinuclear clearing of the sarcoplasm. Some myofiber disarray was definitely observed, and the interstitium lacked collagen deposits. The findings were compatible with the clinical diagnosis of hypertrophic cardiomyopathy. The lung sections displayed diffuse alveolar damage, with widespread formation of hyaline membranes. Also, microscopic foci of bacterial pneumonia were present. The serological search for causative agents of atypical pneumonia, such as CMV, Mycoplasma, RSV, EBV, HSV1, HSV2, Parvo B19, and Pneumocystis jiroveci proved negative and, therefore, the alveolar damage was viewed as a consequence of the severe nephrotic syndrome and cardiomyopathy-induced pulmonary hypertension. A histological evaluation of other organs and tissues did not reveal any abnormality. 


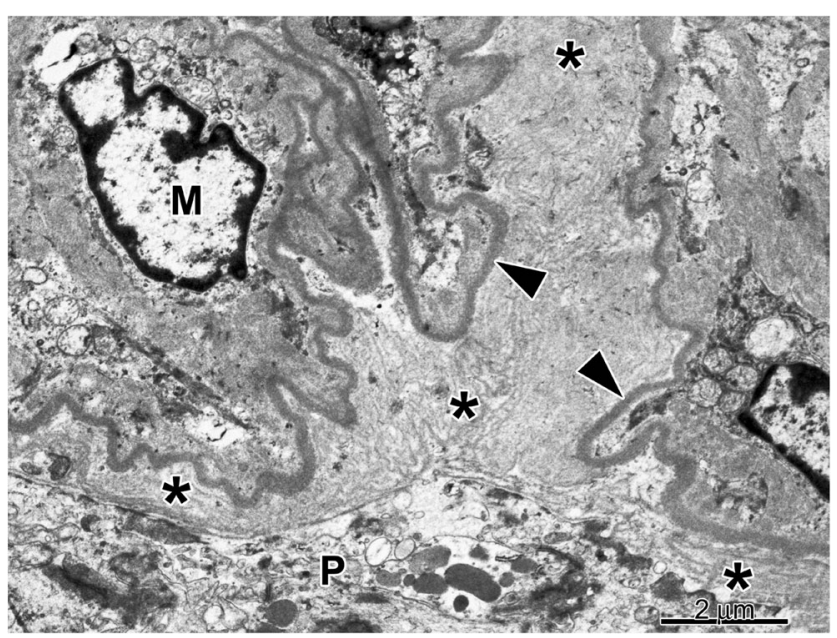

Fig. 4 Portion of a solidified glomerular segment observed using electron microscopy. Several layers of basement membrane material (asterisks) were deposited between the lamina densa of the glomerular basement membrane (arrowhead) and the hypertrophied podocyte $(P)$. The widening of the subendothelial space was caused by the accumulation of extracellular matrix material. $M$ mesangial cell, $\times 2,500$

\section{Discussion}

Pathogenic mutations in mitochondrial disease may occur in the nuclear genes or the mitochondrial genes [14]. The $\mathrm{CoQ}_{10}$ biosynthesis pathway is encoded by nuclear genes. An early diagnosis is crucial because an oral administration with highdose $\mathrm{CoQ}_{10}$ can improve the clinical symptoms. However, obtaining a clear diagnosis is not easy and necessitates biochemical and/or genetic studies. A biochemical diagnosis of $\mathrm{CoQ}_{10}$ deficiency can be established by measuring the $\mathrm{CoQ}_{10}$ content in cultured skin fibroblasts or skeletal muscle biopsies [7]. Usually, routine morphological studies on muscle sections do not yield specific findings; we had a similar experience with our patient during an evaluation of the skeletal muscle samples. Although deafness and retinitis pigmentosa were suspected clinically, a detailed neuropathological examination of the central nervous system did not reveal any significant alterations. The definite diagnosis in our patient, namely that a PDSS2 mutation was the ultimate cause of the $\mathrm{CoQ}_{10}$ deficiency, was achieved via the Illumina TruSight One clinicalexome sequencing and by applying our in-house joint-analysis algorithm, which together revealed two hitherto unknown mutations located within the polyprenyl synthetase domain. It should be mentioned here that large-scale deletions can easily be overlooked by standard mutation screening protocols as the size of the deletion - which was in our case $2923 \mathrm{bp}$ - is more than ten times larger than the reading length $(2 \times 150 \mathrm{bp})$ and most of the bed coordinate-based algorithms used in exome kits filter out deletions starting in the deep intronic regions because exome sequencing only focuses on the exons and short intronic (splicing) regions around the exons.
Regarding the features of PDSS2 mutations published so far, the analysis in an infant with the Leigh syndrome and nephrotic syndrome performed by López et al. [7] identified a heterozygous $\mathrm{C} / \mathrm{T}$ transition at nucleotide 964 , changing amino acid 322 from glutamine to a stop codon and a heterozygous $\mathrm{C} / \mathrm{T}$ transition at nucleotide 1145 , changing amino acid 382 from serine to leucine in the seventh conserved domain in transprenyl diphosphate synthase. In the study by Sadowski et al. on the single-gene cause of the steroidresistant nephrotic syndrome in 1,783 unrelated families, 2 infants had PDSS2 homozygous mutations (c.1145C > T, p.Ser382Leu and c.1151C > A, p.Ala384Asp) [8].

Interestingly, $P D S S 1$-mutant patients seem to have a different clinical presentation than those with PDSS2 mutations, even though both mutations cause a transprenyltransferase deficiency. Mollet et al. evaluated a boy and his sister affected by $\mathrm{COQ}_{10}$ deficiency [15]. They were born from the marriage of first cousins. Both were normal at birth, but then developed deafness, optic atrophy, obesity, macrocephaly, livedo reticularis, cardiac valvulopathy, and mild mental retardation, and both had a prolonged lifespan. Lactate in plasma was mildly elevated. Their disease was attributed to a homozygous missense mutation in PDSS1 (c.977 T > G, p.Asp308Glu). Vasta et al. performed targeted next-generation sequencing on 26 patients with known or suspected mitochondrial disorders [16]. An infant girl with $\mathrm{COQ}_{10}$ deficiency, developmental delay, nephrotic syndrome, leukoencephalopathy, lactate peak, and failure to thrive was found to suffer from a PDSSI mutation characterized by a compound heterozygote with two novel variants in prenyl (decaprenyl) diphosphate synthase, subunit 1 (c. $661 \mathrm{C}>\mathrm{CT}$; p.Arg221Term and c.1108A > AC; p.Ser370Arg). The kidney biopsy revealed acute tubular epithelial damage. The patient died at 16 months of age because of renal failure.

In our patient, the negativity of the glomeruli on immunofluorescence, along with the focal segmental collapse of glomerular capillary tufts and enlarged, proliferating podocytes covering the consolidated areas suggested two possibilities, namely DMS or collapsing glomerulopathy as the cause of the infantile-onset nephrotic syndrome. DMS was concluded based on three findings. First, the combination of fetal-looking glomeruli and more matured glomeruli exhibiting three types of lesions was similar to that observed in glomerular disorders associated with the mutations of the Wilms' tumor suppressor gene, e.g., Denys-Drash syndrome and isolated DMS [17]; however, in our case, the deepest glomeruli were the most severely affected. Second, an evaluation under electron microscopy of glomeruli demonstrated the lamellation of the GBM, which has been regarded as the distinctive ultrastructural lesion of DMS [18]. Third, the aberrant AE1/AE3 expression of podocytes and parietal epithelial cells, characteristic of primary focal-segmental glomerulosclerosis, including the collapsing variant, was not encountered at all [19]. 
During nephrogenesis, the podocytes differentiate from an epithelial to a mesenchymal phenotype, characterized by the expression of mature podocyte markers, such as the WT-1 protein, the disappearance of expression of desmosomal proteins, cytokeratin and PAX2, and the loss of proliferation markers [20]. Studies on podocyte proliferation and phenotype in Denys-Drash syndrome, isolated DMS, collapsing glomerulopathy, and HIV-associated nephropathy have revealed that in these disorders the podocytes re-express proliferation markers, and become focally negative for WT-1 and positive for PAX2 [17, 21, 22]. A similar dysregulation of the podocyte phenotype and proliferation was noted in our patient, suggesting that whatever the etiology, a common phenotypic response occurs in podocytopathies with collapsing lesions. Nevertheless, the lesions of DMS per se did not seem to be responsible for the rapid deterioration in the renal function of our patient that culminated in anuria.

A morphologically similar glomerular disease called collapsing glomerulopathy developed in $k d / k d$ mice with a $P d s s 2$ gene mutation [21]. The $k d / k d$ mouse model of collapsing glomerulopathy led Gasser et al. to hypothesize that human PDSS2 polymorphism might be associated with podocyte diseases [23]. The authors genotyped 9 single-nucleotide polymorphisms in the PDSS2 gene in 377 patients with primary focal segmental glomerulosclerosis. Among EuropeanAmerican patients, a pair of proxy single-nucleotide polymorphisms was significantly associated with podocyte disease, and patients homozygous for one PDSS2 haplotype had a greatly increased risk of podocyte disease. In contrast, the distribution of PDSS2 genotypes and haplotypes was similar in Afro-American patients and controls. Thus, a PDSS2 haplotype is associated with an increased risk for focal segmental glomerulosclerosis in European-Americans.

\section{Conclusion}

Our case presentation extended medical knowledge on the clinicopathological spectrum of PDSS2 mutation-induced podocyte mitochondriopathy, and described two new pathogenic mutations in the PDSS2 gene that both affected the polyprenyl synthetase domain. The mutations lead to infantile-onset multisystemic disease. Regrettably, the multiorgan dysfunction in our patient did not show any signs of improvement following the administration of high-dose $\mathrm{CoQ}_{10}$. We do not know whether the failure of treatment was due to the poor bioavailability of the $\mathrm{CoQ}_{10}$ that was administered, or whether other disturbed physiological functions of $\mathrm{CoQ}_{10}$ played a part in the progressive nature of the disease. Based on the findings of the genetic analysis presented above, a prenatal genetic test was offered within the framework of genetic counseling to this couple. An analysis of the fetal DNA obtained from a chorionic villus biopsy detected only the maternal PDSS2 mutation. The carrier baby, in compliance with the inheritance model of PDSS2, was born healthy.

Acknowledgements We would like to thank the patient's family for their collaboration and Mrs E Borzási, Mrs M Mustoha and Mrs T Vida-Szücs for their expert assistance with the laboratory work. We are very grateful to our reviewers for their valuable comments and opinions.

Funding This study was supported by the GINOP-2.3.2-15-2 grant (TK, ZM) provided by The National Research, Development and Innovation Office (Hungary). The state funders played no role in the study design, data collection and analysis, decision to publish, or in the preparation of the manuscript.

Compliance with ethical standards The clinical investigation was conducted in accordance with the principles expressed in the Declaration of Helsinki. Written informed consent was obtained from all participants involved in the study.

Conflicts of interest The authors declared that no competing interests exist. All the authors declare that the results presented here have not been published previously in whole or part, except in an abstract format.

\section{References}

1. Trevisson E, Dimauro S, Navas P, Salviati L (2011) Coenzyme Q deficiency in muscle. Curr Opin Neurol 24:449-456

2. Desbats MA, Vetro A, Limongelli I, Chretien D, Kadhom N, Edery P, Lebideau M, Dallner G, Munnich A, Ernster L, Rustin P (2015) Primary coenzyme Q10 deficiency presenting as fatal neonatal multiorgan failure. Eur J Hum Genet 23:1-5

3. Desbats MA, Lunardi G, Doimo M, Trevisson E, Salviati L (2014) Genetic bases and clinical manifestations of coenzyme Q10 (CoQ10) deficiency. J Inherit Metab Dis 10:145-156

4. Acosta MJ, Vazquez Fonseca L, Desbats MA, Cerqua C, Zordan R, Trevisson E, Salviati L (2016) Coenzyme Q biosynthesis in health and disease. Biochim Biophys Acta Bioenerg 1857(8):1079-1085

5. Rötig A, Appelkvist EL, Geromel V, Chretien D, Kadhom N, Edery P, Lebideau M, Dallner G, Munnich A, Ernster L, Rustin P (2000) Quinone-responsive multiple respiratory-chain dysfunction due to widespread coenzyme Q10 deficiency. Lancet 356:391-395

6. Rahman S, Clarke CF, Hirano M (2012) 176th ENMC International Workshop: diagnosis and treatment of coenzyme Q10 deficiency. Neuromuscul Disord 22:76-86

7. López LC, Schuelke M, Quinzii CM, Kanki T, Rodenburg RJ, Naini A, Dimauro S, Hirano M (2006) Leigh syndrome with nephropathy and CoQ10 deficiency due to decaprenyl diphosphate synthase subunit 2 (PDSS2) mutations. Am J Hum Genet 79: $1125-1129$

8. Sadowski CE, Lovric S, Ashraf S, Pabst WL, Gee HY, Kohl S, Engelmann S, Vega-Warner V, Fang H, Halbritter J, Somers MJ, Tan W, Shril S, Fessi I, Lifton RP, Bockenhauer D, El-Desoky S, Kari JA, Zenker M, Kemper MJ, Mueller D, Fathy HM, Soliman NA, SRNS Study Group, Hildebrandt F (2015) A single-gene cause in $29.5 \%$ of cases of steroid-resistant nephrotic syndrome. J Am Soc Nephrol 26:1279-1289

9. Kumar P, Henikoff S, Ng PC (2009) Predicting the effects of coding non-synonymous variants on protein function using the SIFT algorithm. Nat Protoc 4:1073-1081

10. Adzhubei IA, Schmidt S, Peshkin L, Ramensky VE, Gerasimova A, Bork P, Kondrashov AS, Sunyaev SR (2010) A method and 
server for predicting damaging missense mutations. Nat Methods 7: 248-249

11. Siepel A, Pollard KS and Haussler D (2006) New methods for detecting lineage-specific selection. In: Apostolico A, Guerra C, Istrail S, Pevzner PA, Waterman M (eds) Research in computational molecular biology. RECOMB 2006. Lecture Notes in Computer Science, vol 3909. Springer, Berlin, Heidelberg

12. Li H, Handsaker B, Wysoker A, Fennell T, Ruan J, Homer N, Marth G, Abecasis G, Durbin R, 1000 Genome Project Data Processing Subgroup (2009) The sequence alignment/map format and SAMtools. Bioinformatics 25:2078-2079

13. Lek M, Karczewski KJ, Minikel EV et al (2016) Analysis of protein-coding genetic variation in 60,706 humans. Nature 536: 285-291

14. Rahman SHA (2013) Mitochondrial disease - an important cause of end-stage renal failure. Pediatr Nephrol 28:357-361

15. Mollet J, Giurgea I, Schlemmer D, Dallner G, Chretien D, Delahodde A, Bacq D, de Lonlay P, Munnich A, Rötig A (2007) Prenyldiphosphate synthase, subunit 1 (PDSS1) and $\mathrm{OH}$-benzoate polyprenyltransferase (COQ2) mutations in ubiquinone deficiency and oxidative phosphorylation disorders. J Clin Invest 117:765772

16. Vasta V, Merritt JL, Saneto RP, Hahn SH (2012) Next-generation sequencing for mitochondrial diseases: a wide diagnostic spectrum. Pediatr Int 54:585-601
17. Yang Y, Jeanpierre C, Dressler GR, Lacoste M, Niaudet P, Gubler MC (1999) WT1 and PAX-2 podocyte expression in Denys-Drash syndrome and isolated diffuse mesangial sclerosis. Am J Pathol 154:181-192

18. Stokes MB, Markowitz GS, D'Agati VD (2017) Glomerular diseases associated with nephrotic syndrome and proteinuria. In: Zhou XJ, Laszik ZG, Nadasdy T, D'Agati VD (eds) Silva's diagnostic renal pathology, 2nd edn. Cambridge University Press, Cambridge, pp 130-132

19. Bariéty J, Bruneval P, Hill G, Irinopoulou T, Mandet C, Meyrier A (2001) Posttransplantation relapse of FSGS is characterized by glomerular epithelial cell transdifferentiation. J Am Soc Nephrol 12: 261-274

20. Bariéty J, Bruneval P, Hill GS, Mandet C, Jacquot C, Meyrier A (2003) Transdifferentiation of epithelial glomerular cells. J Am Soc Nephrol 14(Suppl 1):S42-S47

21. Barisoni L (2005) The $\mathrm{kd} / \mathrm{kd}$ mouse Is a model of collapsing glomerulopathy. J Am Soc Nephrol 16:2847-2851

22. Yang AH, Chen JY, Chen BF (2004) The dysregulated glomerular cell growth in Denys-Drash syndrome. Virchows Arch 445:305314

23. Gasser DL, Winkler CA, Peng M, An P, McKenzie LM, Kirk GD, Shi Y, Xie LX, Marbois BN, Clarke CF, Kopp JB (2013) Focal segmental glomerulosclerosis is associated with a PDSS2 haplotype and, independently, with a decreased content of coenzyme Q10. Am J Physiol Renal Physiol 305:F1228-F1238 